\title{
RADIO EMISSION FROM CLOSE BINARY SYSTEMS AND NOVAE
}

R.M. Hjellming

National Radio Astronomy Observatory

Socorro, New Mexico, 87801, U.S.A.

This review of the radio emission properties of close binary systems and novae will be partly concerned with surveying the star systems that exhibit continuum radio emission, and partly concerned with discussing the implications of the observed radio emission. The phenomena we will encounter will range from purely thermal continuum radio emission for the case of ionized nova shells to strong, non-thermal continuum radio emission produced by relativistic particles in both 'normal' and $\mathrm{x}$-ray emitting close binary systems. In keeping with the subject of this symposium, let me emphasize that all regions involving radio emission are from the portions of the stellar enviromment where $\mathrm{N}_{\mathrm{e}} \leq 10^{10} \mathrm{~cm}^{-3}$, generally rather high in the stellar or systemic atmosphere; however, events more closely associated with the stars themselves are the root causes of the phenomena, supplying energy, mass, magnetic fields, and relativistic particles.

NOVAE

Because the physics of the radio emission is simplest for the case of nova shells, let us begin with a brief discussion of the three novae for which light curves at radio wavelengths are available, and for which the radio emission mechanism is thermal. The first detected radio novae were Nova HR Delphini 1967 and Nova FH Serpentis 1970, first detected in 1970 (Hjellming and Wade, 1970). Unfortunately. HR Del was first searched for radio emission 3 years after initial optical outburst; therefore the radio variations seen in Fig. 1 constitute only the last portion of the life of a radio nova, when the nova shell is evolving from an optically thick state at radio wavelengths to the flat spectrum of an optically thin, dilute, ionized gas.

With $\mathrm{FH}$ Ser the timing of the initial detection was much more propitious and almost all of the interesting stages of this radio nova were seen as shown in Fig. 1 . In FH Ser we see both the initial rise of the radio flux due to an expanding, optically thick source and the complete transition to an optically thin source as the ionized gas density decreases and the optical depth at radio wavelengths becomes small.

The general expression for the radio flux density for thermal bremstrahlung emission from an ionized plasma can be written as

$$
s_{v}=\left(2 k / \lambda^{2}\right) \iint T_{e} \exp \left(-\tau_{v}\right) d \tau_{v} d \Omega_{s}
$$


where $k=$ Boltzmann constant, $\lambda$ is the wavelength corresponding to frequency $v, T_{e}$ is the electron temperature, $\tau_{v}$ is the optical depth for which an infinitesimal element can be approximated by

$$
d \tau_{\nu} \cong 0.08235 \mathrm{~T}^{-1.35} v(\mathrm{GHz})^{-2.1} \mathrm{dE}
$$

where $\mathrm{E}$ is the emission measure in units of $\mathrm{pc} \mathrm{cm}^{-6}$, and $\Omega_{\mathrm{s}}$ is the solid angle of the emitting regions.

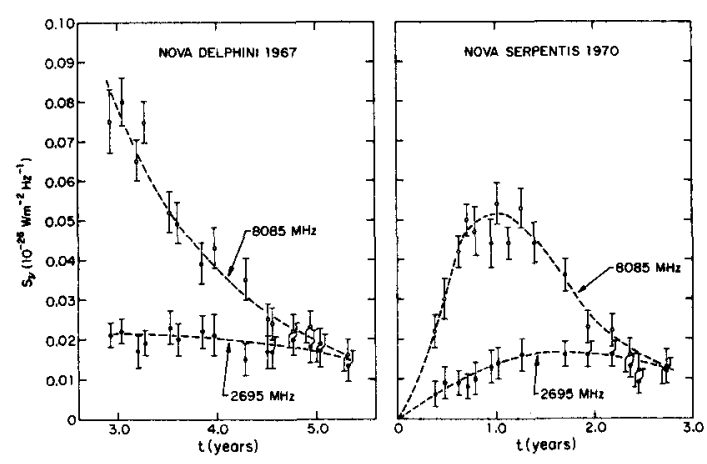

Fig. 1. Radio light curves of Nova HR Delphini 1967 and Nova FH Serpentis 1970 at 2695 and $8085 \mathrm{MHz}$.

From eqns. (1) and (2) we see that in the optically thin state

$$
\mathbf{s}_{v}=\left(2 \mathbf{k} / \lambda^{2}\right)<\mathrm{T}_{\mathrm{e}}^{\Omega} \mathbf{s}^{>}
$$

therefore, since $\mathrm{T}_{\mathrm{e}}$ is generally known to be roughly $10000 \mathrm{~K}$, the early stages of a radio novae give a nearly direct measurement of the growth of the solid angle of the emitting regions. Empirically,

$$
s_{v} \cong\left\{\begin{array}{l}
0.010 \\
0.041
\end{array}\right\}\left(t-t_{0}\right)^{0.5} \text { at }\left\{\begin{array}{l}
2696 \\
8085
\end{array}\right\} \mathrm{MHz}
$$

for the optically thick phase of $\mathrm{FH}$ ser. This means $\left\langle\mathrm{T} \mathrm{e}_{\mathrm{s}}\right\rangle_{8085} /\left\langle\mathrm{T} \mathrm{e}_{\mathrm{s}}\right\rangle_{2695} \cong 0.46$; therefore it is clear that nova shells are complex structures. We can express $\Omega_{\mathrm{s}}$ 
in square arcsec and eqn. (4) becomes

$$
\Omega_{s}=\left\{\begin{array}{l}
0.44^{\prime \prime} \times 0.44^{\prime \prime} \\
0.29^{\prime \prime} \times 0.29^{\prime \prime}
\end{array}\right\}\left(t-t_{0}\right) 0.5\left(10000 / T_{e}\right) \text { at }\left\{\begin{array}{l}
2695 \\
8085
\end{array}\right\} M H z
$$

predicting, for example, a size scale of 0.8 " in 1977.

As seen from Fig. 1, FH ser peaked out at roughly 1 year after outburst for $8085 \mathrm{MHz}$ and somewhat later for $2695 \mathrm{MHz}$. At 2.5 years after outburst it was in an optically thin state as expected for a dilute plasma. This contrasts with HR Del taking 5 years to reach this state. At this point the excitation parameter, given by

$$
\left\langle\mathrm { N } ^ { 2 } \mathrm { v>l/3 } \cong 1 4 \left[\mathrm{d}(\mathrm{kpc})^{2} \mathrm{~s}_{\mathrm{v}}\left(\mathrm{T} \mathrm{e}^{/ 10000)^{-0.5}}\right]^{1 / 3} \mathrm{pc} \mathrm{cm^{-2 }}\right.\right.
$$

was 2.5 and $3.3 \mathrm{pc} \mathrm{cm}^{-2}$, respectively, for $\mathrm{FH}$ Ser and HR Del, assuming distances of 0.7 and $0.8 \mathrm{kpc}$.

The radio light curve of Nova VI500 Cygni (Hjellming 1978) is shown in Fig. 2.

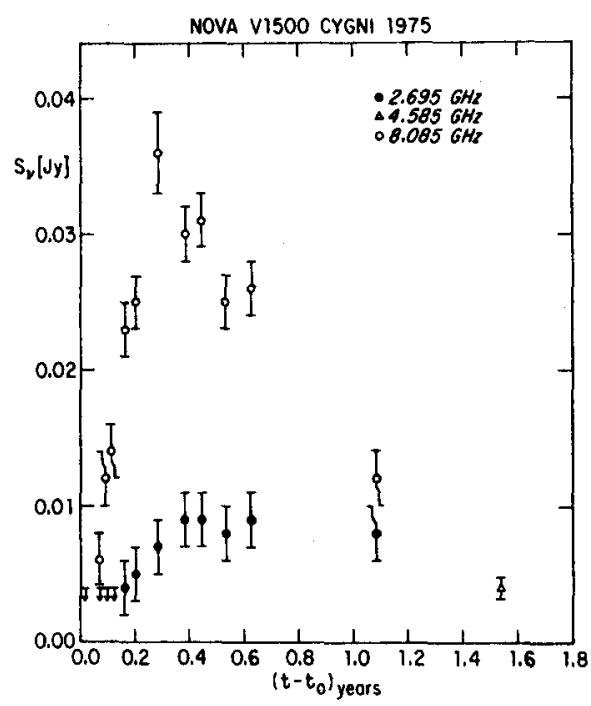

Fig. 2. Radio variations of Nova VI500 Cygni at 2695,4585 , and 8085 MHz plotted as a function of time since initial outburst. 
Because we have been looking for more good cases since 1970, the early history of Nova V1500 Cygni as a radio source was obtained in detail. For the first four weeks after outburst, the radio source had too small a solid angle to be detectable; however, four weeks after outburst it was detected at $8085 \mathrm{MHz}$ and a full light curve was obtained during the next year or so. The last point at $4.585 \mathrm{MHz}$ was obtained with the VLA a couple of months after marginal detections at the 4 mJy level were all that could be obtained at 2695 and $8085 \mathrm{MHz}$ with the NRAO interferometer in Green Bank. Empirically, the first 3-4 months of v1500 Cygni behaved, at these frequencies, as

$$
s_{\nu} \cong\left\{\begin{array}{l}
0.025 \\
0.13
\end{array}\right\}\left(t-t_{0}\right) \text { at }\left\{\begin{array}{l}
2695 \\
8085
\end{array}\right\} \mathrm{MHz}
$$

so we get for the solid angle variations

$$
\Omega_{s} \cong\left\{\begin{array}{l}
0.69^{\prime \prime} \times 0.69 " \\
0.52 " x 0.52 "
\end{array}\right\}\left(t-t_{0}\right)\left(10000 / T_{e}\right) \text { at }\left\{\begin{array}{l}
2695 \\
8085
\end{array}\right\} \mathrm{MHz}
$$

V1500 Cyg apparently reached the optically thin state at radio wavelengths about 1.2 years after initial outburst, at which time its excitation parameter, $<\mathrm{N}^{2} \mathrm{~V}>1 / 3$, was about $3.5 \mathrm{pc} \mathrm{cm}^{-2}$, assuming a distance of $1.4 \mathrm{kpc}$.

The following table summarizes some of the parameters of the three radio novae:

Table 1. Radio Nova Parameters

\begin{tabular}{lcccccc} 
Nova & $S(8085)^{\max }$ & distance & $\Omega_{\mathrm{s}}$ & $\mathrm{N}_{\mathrm{e}}{ }^{2} \mathrm{~V}>\mathrm{l} / 3$ & $\left(\mathrm{t}-\mathrm{t}_{0}\right)$ \\
\hline HR Del 1967 & $>0.08$ & Jy & $0.8 \mathrm{kpc}$ & -- & $3.3 \mathrm{pc} \mathrm{cm}$ & 5 years \\
FH Ser 1970 & 0.045 & 0.7 & $\left(t-\mathrm{t}_{0}\right)$ & 0.5 & 2.5 & 2.5 \\
V1500 CYg 1975 & 0.035 & 1.4 & $\left(t-\mathrm{t}_{0}\right)$ & 3.5 & 1.2
\end{tabular}

In the above table the last two columns correspond to the state where one can take $\tau_{v} \cong 0.1$. combining this and the fact that for all three novae, the excitation parameter is about 3 at this point, one finds that this implies $\mathrm{N} \cong 20-50 \mathrm{~cm}^{-3}$, $\mathrm{v}^{1 / 3} \cong 0.02-0.05 \mathrm{pc}$.

Recent VLA observations have shown that in July 1977, both HR Del and FH Ser were still detectable as point radio sources. FH Ser was $(1.3 \pm 0.4) \mathrm{mJY}$ at $6 \mathrm{~cm}$ and HR Del was $(2 \pm 0.4) \mathrm{mJ}$. These results raise questions about whether these two novae still have unresolved optically thin thermal shells, or whether there might be core stellar wind sources remaining that would be expected to have a spectrum proportional to $v$.

There are roughly 20 stars for which there is strong evidence for association 
with steady thermal radio emission. Although we will not discuss these objects in detail, it is worthwhile to list them, since many are associated with stars that are subjects of discussion in this symposium.

Table 2. Strong, Optically Thick, Thermal Sources

\begin{tabular}{|c|c|c|}
\hline Name & Comment & Reference \\
\hline LkHa 101 & Core with halo & Brown et. al. 1976 \\
\hline MWCl 137 & Core with weak halo & Altenhoff et. al. 1976 \\
\hline M 2-9 & Two compact components & Marsh et. al. 1975 \\
\hline$M 1-23$ & $2 "$ structure & $"$ \\
\hline HD 316248 & 3" structure & $"$ \\
\hline HDI67362 & $1 "$ or less & $"$ \\
\hline RY Sct & structure 3" and less & Hjellming et. al, 1973 \\
\hline MWC957 & $1^{\prime \prime}$ or less & Marsh et. al. 1975 \\
\hline vy $2-2$ & 1" or less & $"$ \\
\hline v1016 Cyg & $1 "$ or less & Altenhoff et. al. 1976 \\
\hline Hb 12 & l" or less & Marsh et. al. 1976 \\
\hline M 1-11 & & Altenhoff et. al. 1976 \\
\hline MWC 349 & 2-3" structure & Baldwin et. al. 1973 \\
\hline$\gamma^{2} \mathrm{VeI}$ & WR binary & Seaquist 1976 \\
\hline
\end{tabular}

Table 3. Weak, Thermal sources

\begin{tabular}{ll} 
Name & \multicolumn{1}{c}{ Reference } \\
\hline AG Peg & Gregory et. al. 1977 \\
V1329 Cyg & Altenhoff and Wendker 1973 \\
R Agr & Gregory and Seaquist 1974 \\
T Tauri & Spencer and Schwartz 1974 \\
P Cyg & Wendker et. al. 1973
\end{tabular}

With the objects in Tables 2 and 3 there is a difficult problem to be faced in distinguishing between stellar wind sources, nova shells, and planetary or protoplanetary nebula phenomena. Up till now most of the very small, optically thick, thermal sources have been interpreted (Wright and Barlow 1975, Panagia and Felli 1975, Marsh et. al. I976) in terms of $\mathrm{R}^{-2}$ density distributions axising from a 
stellar wind with a reasonable mass loss rate. Radio data on novae mainly provide information about nebular shells ejected in relatively brief episodes of mass ejection. An example of an object that may be either nova-like, due to a stellar wind, or a proto-planetary, is HM Sge (Feldman 1977), for which current data is somewhat contradictory.

To close our discussion of nova phenomena at radio wavelengths, and perhaps to begin the discussion of close binary phenomena, we must mention Nova Monocerotis 1975 (A0620-00, Owen et. al. 1976). This is the only optical nova known to exhibit transient $x$-ray emission. In the space of seven days a radio source associated with this object went from $300 \mathrm{mJy}$ down to $50 \mathrm{mJy}$, and a few weeks later was not detectable. So little information is available about the radio emission from this object that little can be said except that it is not at all related to normal nova shell radio phenomena.

\section{CLOSE BINARIES}

If we exclude the stars which are associated with very strong $x-r a y$ emission, there are roughly 16 binary systems that are known to be continuum, non-thermal, radio sources. These 16 are listed in the Table 4:

Table 4. Radio Binaries

\begin{tabular}{|c|c|c|c|c|}
\hline Name & Spectral Types & $\mathrm{d}(\mathrm{pc})$ & $\mathrm{P}$ (days) & Reference \\
\hline$\alpha$ Sco B & $\mathrm{B} 4 \mathrm{~V}+\mathrm{M} 2 \mathrm{I} \mathbf{a}$ & 180 & - & Hjellming \& Wade 1971 \\
\hline B Per Algol & $\mathrm{B} 8+\mathrm{G} 5 \mathrm{III}+\mathrm{A} 7 \mathrm{~m}$ & 25 & 2.9 & Wade \& Hjellming 1972 \\
\hline$\beta$ Lyr & $88 p+?$ & 260 & 12.9 & $"$ \\
\hline b Per & $\mathrm{A} 2 \mathrm{IV}+\mathrm{G}+\mathrm{F} 5 \mathrm{~V}$ & 56 & 1.5 & Hjellming \& Wade 1973 \\
\hline CC Cas & $09 I V+09 I V$ & 1000 & 3.4 & Gibson \& Hjellming 1974 \\
\hline AR LAC & $\mathrm{G} 2 \mathrm{IV}+\mathrm{KOIV}$ & 50 & 2.0 & Hjellming \& Blankenship 1973 \\
\hline UX Ari & G5V + KOIV & 50 & 6.4 & Gibson et. al. 1975a \\
\hline RT Lac & G9IV + KIIV & 200 & 5.1 & Gibson et. al. $1975 \mathrm{~b}$ \\
\hline HR1099 & $G+K 0$ & 35 & 2.8 & Owen et. al. 1977 \\
\hline$\lambda$ And & G6 & 23 & 20.5 & Bath \& Wallerstein 1976 \\
\hline SZ Pse & $F 8 V+K I V-I V$ & 100 & 4.0 & Owen and Gibson 1978 \\
\hline UV Psc & G2 & 125 & 0.9 & Spangler et. al. 1978 \\
\hline 54 Cam & GO & 38 & 11.1 & $"$ \\
\hline$\sigma \operatorname{CrB}$ & $F 6+G l$ & 23 & 1.1 & " \\
\hline HD 216489 & Ko & - & 24.6 & $"$ \\
\hline $\mathrm{HD} 224085$ & KOV & 29 & 6.7 & $"$ \\
\hline
\end{tabular}


The stars in Table 4 are divided into two groups, and are also listed roughly in order of discovery. In the first group, all of which were discovered before any of the second group, the dominant stars are of spectral type $A$ or earlier, whereas for the second group, $F, G$, and $K$ spectral types are found. Since they are listed in order of discovery, Table 4 shows one of the major points of this review - the most frequently fourid continuum radio stars are now of relatively late spectral type.

a Sco B is the B4V visual companion to the M2Ia star known as Antares. While a Sco B itself is not known to be a close binary, its discovery led to the suggestion (Wade and Hjellming 1972) that stellar mass exchange and the binary phenomena are essential ingredients in this type of radio star emission. Following this suggestion, $B$ Per (Algol) and B Lyr were soon discovered as radio sources and, indeed, with the exception of a few reports of radio emission from single stars like Betelguese (Wendker et. al. 1973), all variable radio stars found since have been binary or multiple star systems. One might worry about observational selection effects; however, a recent search of nearby single stars by Gibson (1977) using the VLA has resulted in no detections. In any case, the hypothesis that continuum radio star emission occurs dominantly in binary star systems has proven so fruitful over the years that until contrary evidence is found, it can be considered to be the rule.

Amongst the first five radio binaries in Table 4, only $B$ Per (Algol) has shown high radio flux levels and a spectacular range of behavior. There is some suggestion for cycles of radio behavior as seen in Fig. 3 .

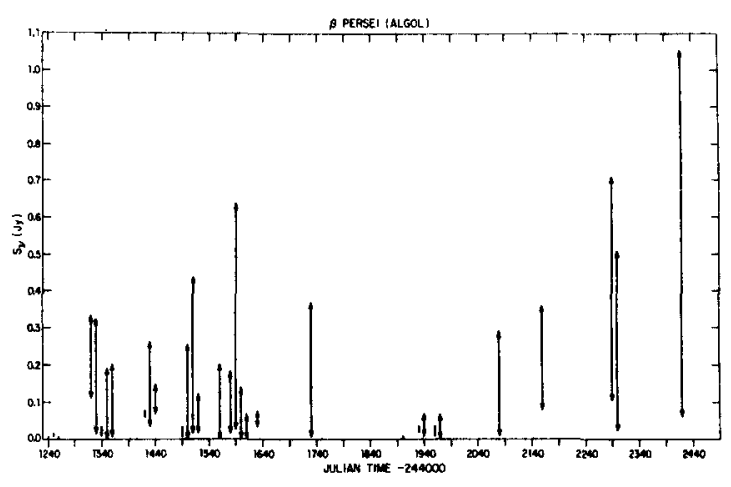

Fig. 3. A sumary of the range of radio continuum flaring in Algol during 1971-1975.

When first detected in 1972 Algol was a very weak radio source, but in January 1972 it was found to be variable by a factor of ten or more in several hours. 
Because of crowding, only the widest range of events are shown in Fig. 3 . In Fig. 4 we show six Algol radio events that show both typical and unique behavior.

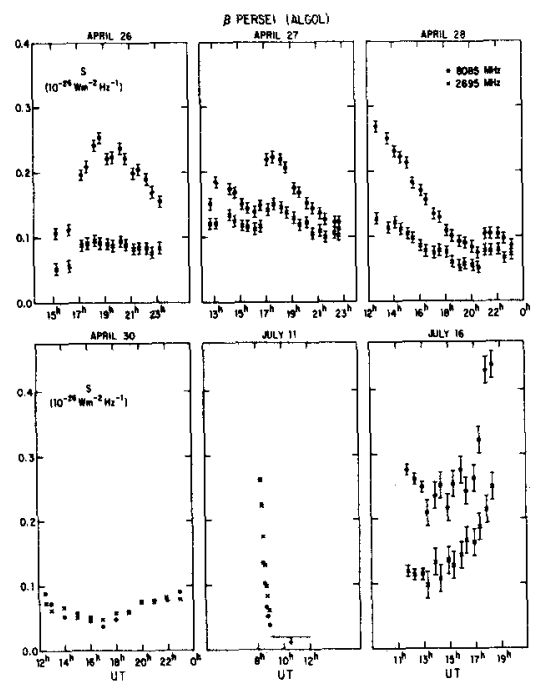

Fig. 4. Six Algol events at 2695 and $8085 \mathrm{MHz}$ seen in 1972

In Fig. 4, the April 26, April 27, April 28, and July 16, 1972 events are typical of the segments of events that one observes when Algol is flaring. In the April 26-28 events three of the clearest cases of abrupt discontinuities, or upward jumps in radio flux, are found. The April 30 behavior is typical of the extremely flat spectrum behavior Algol shows a great deal of the time. However, the July 11, 1972 event is unique in showing a decaying non-thermal spectrum and exceptionally short time scale. Fortunately, a large portion of this event was also observed by pooley and Ryle (1973), and the combined data are shown in Fig. 5.

The July 11 event reached a peak of $0.65 \mathrm{Jy}$ at $6 \mathrm{~cm}$, had a complete lifetime of 2 hours, and ended with a non-thermal decay. This contrasts with normal behavior where event lifetimes are typically more like 16 hours (Hjellming 1972). When the peak of an Algol event is caught, the event looks very much like the event shown in Fig. 6. Frequently double peaks are found in the higher frequency data for Algol flare events. 


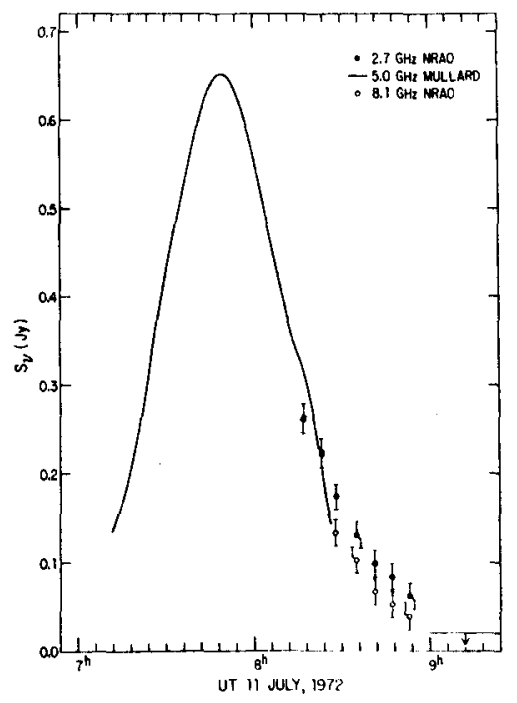

Fig. 5. The brief, non-thermal event of July 11, 1972 in Algol as measured at $3.7,6$, and $11 \mathrm{~cm}$

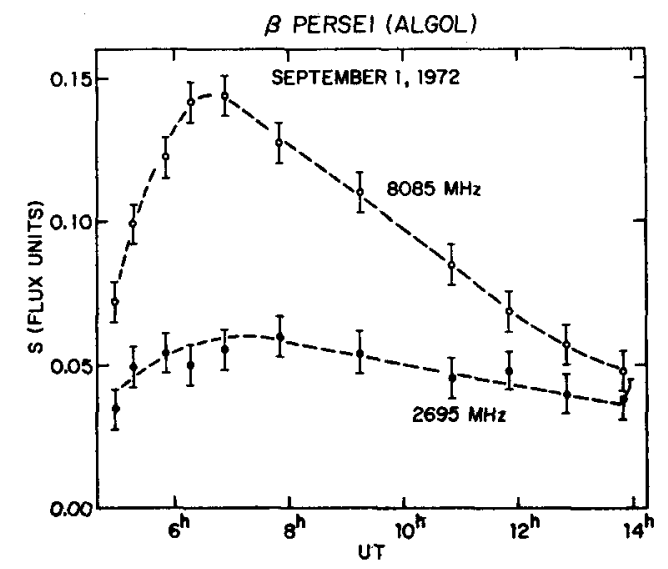

Fig. 6. A "typical" Algol event observed at 2695 and $8085 \mathrm{MHz}$ on September 1, 1972 
Algol as has three unique characteristics amongst continuum radio stars: (1) it has flared to very high levels ( > $1 \mathrm{Jy})$; (2) it has been found to be a weak $\mathrm{X}$-ray source (Schnopper et. al. 1976); and (3) the size of the radio emitting regions has been measured and observed to grow in time in a few hours.

On May 4-5, 1974 a $0.7 \mathrm{Jy}$ event was found by B.G. Clark et. al. (1975) to have a size of 0.004 " (or $0.1 \mathrm{AU}$ ) with an apparent brightness temperature of $4 \times 10^{8} \circ \mathrm{K}$. Several months later T. Clark et. al. (1976) observed a 1 Jy event on January 16 , 1975 which showed a complex source with a size scale of $0.0018 "$, corresponding to $0.045 \mathrm{AU}$, with brightness temperatures of $1010 \circ \mathrm{K}$. Remembering that the close double in the Algol system has a semi-major axis of $0.066 \mathrm{AU}$, and the diameter of both stars is $0.03 \mathrm{AU}$, we see that on one occasion the Algol radio source has been seen to be larger than the stellar disks, and on another occasion 50 larger than the semi-major axis. In addition, the very high brightness temperatures confirm the evidence of the occasionally occurring non-thermal spectrum that the Algol radio phenomena is non-thermal and probably due to synchrotron radiation from relativistic electrons. In the May 4-5, 1975 event, the data are consistent with $5 \times 10^{34}$ ergs in relativistic electrons and $4 \times 10^{34}$ ergs in magnetic fields of roughly 1 Gauss strength.

The greatest puzzle of the Algol radio source and, indeed, all the other variable radio stars is how and where in the binary system are these relativistic particles produced and accelerated to the $10 \gamma$ level or more. To put this in perspective, one should remember that in binary radio stars the level of radio emission and the energies in particles are $10^{5}-10^{11}$ times that for the largest radio event ever seen on the Sun, with $10^{6}$ being the typical factor for Algol events.

Now let us consider the radio binaries with relatively late spectral types. After AR Lac was discovered (see Table 4) while searching a group of close binaries with considerable period changes, Doug Hall (1973) suggested that because of certain similarities UX Ari should be a radio source. It was found to be one by Gibson and Hjellming (1974). Following various discussions with Hall about how AR Lac and UX Ari were members of the so-called RS CVn class of binaries (Hall 1965), Gibson (1975) developed in his thesis the idea that these systems might frequently be continuum radio emitters. This hypothesis has since resulted in the detection of RT Lac (Gibson et. al. 1975b), HR 1099 (Owen et. al. 1977), and a number of other RS CVn or RS CVn-like binaries as seen in Table 1. Hall (1975) defined the RS CVn class in terms of: (1) periods from 1 day to 14 days; (2) hotter component of spectral type F-G and luminosity class IV-V; and (3) strong $H$ and $K$ emission seen in the spectrum outside eclipse. CurrentIY UV Psc, $\lambda$ And, and HD 216486 violate the period definition, but are discussed by Hall (1975) as short and long period relatives of the $\mathrm{RS}$ CVn class.

of particular importance is the fact that the Radio emission from HRl099 has been found on occasion to be highly circularly polarized (Owen et. al. 1977). This 
has been interpreted by owen et. al. (1977) in terms of gyro-synchrotron radiation. Thus it is clear that the energetic electrons produced in binary star systems range from the mildly relativistic to the highly relativistic $(>10 \mathrm{\gamma})$.

STRONG X-RAY EMITTING BINARIES

There is a group of close binaries that exhibit variable, non-thermal, continuum radio emission that are primarily defined by the fact that they are very strong $\mathrm{X}$-ray sources. The five objects in this class are listed in Table 5 .

Table 5. Strong X-Ray and Radio Emitting Close Binaries

\begin{tabular}{llc} 
Name & \multicolumn{1}{c}{ Star system } & Period \\
\hline Sco $x-1$ & blue star, emission lines & 0.787 days \\
Cyg X-3 & variable IR binary star & 4.8 hours \\
Cyg X-1 & 09.5 Iab + Black Hole (?) & 5.6 days \\
Cyg X-2 & G(subdwarf?), emission lines & - \\
GX17+2 & G Dwarf
\end{tabular}

of the objects in Table 5, sco $x-1$, cyg $x-1$, Cyg $x-2$, and $6 x 17+2$ have radio characteristics similar to some of the radio binaries (Hjellming 1975). However, Cyg $x-3$ is a spectacular exception in that it occasionally shows quasar-like behavior by flaring from normal quiescient levels of about $0.1 \mathrm{Jy}$ to 10-20 Jy. with energetics $10^{-9}$ that of typical quasars (Hjellming 1973) and physical characteristics nearly identical to the expanding, synchrotron radiating cloud theory originally conceived to explain quasar radio emission, Cyg X-3 can at least be considered as a nano-quasar.

For both normal and $x$-ray binaries that emit variable continuum radio emission, the major unsolved problem is the origin and acceleration of relativistic electrons.

The National Radio Astronomy Observatory is operated by Assoclated universities Inc., under contract with the U.S. National Sçience Foundation.

REFERENCES

Altenhoff, W.J., Braes, L.L.E., Olnon, F.M., Wendker, H.J. 1976, Astron. and

Astrophys., 46, 11.

Altenhoff, W.J. and Wendker, H.J. 1973, Nature, 241, 37.

Baldwin et. al. 1975, 1973, Nature, 241, 38 .

Bath. G.T. and Wallerstein, G. 1975, P.A.S.P., 88, 759.

Brown, R.L. et. al. 1976, M.N.R.A.S., 175, 87P. 
Clark, B.G., Kellermann, K.I., and Shaffer, D. 1975, Astrophy. J., 198, L123.

Clark, T.A., Hutton, L.K., Ma, C., Webster, W., Hinteregger, C.A., Knight, C.A., Rogers, A.E.E., Whitney, A.E., Shapiro, I.I., Wittels, J.J., Niell, A.E., and Resch, G.M. 1976, Astrophy. J., 206, L107.

Feldman, P.A. 1977, IAU Symposium on Planetary Nebulae, Ithaca, N.Y.

Gibson, D.M. 1975, Ph.D. thesis, U. of Virginia.

Gibson, D.M. 1977 (in preparation).

Gibson, D.M. and Hjellming, R.M. 1974, P.A.S.P., 86, 652 .

Gibson, D.M., kjellming, R.M., and Owen, F.N. 1975a, Astrophy. J., 200, L99.

Gibson, D.M., Hjellming, R.M., and Owen, F.N. 1975b, IAU Circ. No. 2789.

Gregory, P.C. and Seaquist, E.R. 1974, Nature, 247, 532 .

Gregory, P.C., Kwok, S., and Seaquist, E.R. 1977, Ap.J.. 211, 429.

Hal1, D. 1973, private communication.

Hall, D. 1975, IAU Collog. No. 29 (in press).

Hjellming, R.M. 1972, Nature Phy. Sci., 238, 52.

Hjellming, R.M. 1973, Science, 182, 1089 .

Hjellming, R.M. 1975, "Physics of Non-Thermal Radio Sources", ed. G. Setti (Dordrecht: Reide1), p. 203.

Hjellming, R.M. 1978, Astron. J. (to be submitted).

Hjellming, R.M. and Blankenship, L.C. 1973, Nature Phy. Sci., 243, 81.

Hjellming, R.M., Blankenship, L.C., and Balick, B. 1973, Nature Phy. Sci., 242, 84.

Hjellming, R.M. and Wade, C.M. 1970, AP. J. (Letters), 162, L1.

Hjellming, R.M. and wade, C.M. 1971, Astrophy. J., 168, L115.

Hjellming, R.M. and Wade, C.M. 1973, Nature, 242, 250.

Marsh, K.A., Purton, C.R., Feldman, P.A. 1976, Astron. and Astrophys., 49, 211.

Owen, F.N., Balonek, T.J., Dickel, J., Terzian, Y., and Gottesmann, S.T., 1976, Astrophy. J., 203, L15.

Owen, F.N. and Gibson, D.M. 1978 (in preparation).

Owen, F.N., Jones, T.W., and Gibson, D.M. 1977, Astrophy. J., 210, L27.

Panagia, N. and Felli, M. 1975, Astron. Ap., 39, 1.

Pooley, G.G. and Ryle, M. 1973, Nature, 244, 270.

Seaquist, E.R., 1976, Astrophy. I., 203, I.35.

Schnopper, H.W., Delvaille, J.P., Epstein, A., Helmken, H., Murray, S.S., Clark, G., Jernigan, G., and Doxsey, R. 1976, Astrophys. J., 210, L75.

Spangler, S.R., Owen, F.N., and Hulse, R.A. 1978, A.J. (in press).

Spencer, J.H. and Schwartz, P.R. 1974, Astrophy. J., 188, L105.

Wade, C.M. and Hjellming, R.M. 1972, Nature, 235, 270 .

Wendker, H.J., Baars, J.W.M., and Altenhoff, W.J. 1973, Nature Phy. Sci.., 245, 118. Wright, A.E. and Barlow, M.J., 1975, M.N.R.A.S., 170, 41 .

\section{I S C US S I ON of paper by HJELLMING:}

FITZGERALD: You said that HM Sge had a very peculiar spectrum, can you give us a sketch of its radio spectrum?

HJELLMING: Not convincingly, the problem is it has been reported to be variable at $21 \mathrm{~cm}$, but not at higher frequencies.

RUCINSKY: Do you see any difference in the radio flaring activity between Algol and the RS CVn-type binaries?

HJELLMING: Basically no, with the possible exception that the RS CVn-type binaries more often show optically thin, non-thermal characteristics in decaying events.

DE LOORE: Can you discriminate between mass loss and mass transfer in your radio observations? 
HJELLMING: No. We see only the effects of relativistic electrons and magnetic fields, and speculate that the events must have something to do with mass loss and/or mass transfer; however, there is no established connection between continuum radio flares and mass transfer or mass loss.

BIERMANN: You said that it was not reasonable to compare the radio flares of Algol with those of the Sun. Is there any radio source you would prefer to compare with Algol?

HJELLMING: Yes, quasars and nano-quasars.

KRAFT: $\lambda$ And has very strong CaII emission and is a binary with a period of about 20 days. Is CaII emission strength in binaries correlated with the presence of radio emission, and if so, would you find that as the period increases, the radio emission disappears?

HJELLMING: The answer to both questions must be that there is no significant statistical evidence for such correlations or lack of correlations. However, the CaII emission is one of the defining characteristics of RS CVn-type stars.

WILSON: Two comments. First, the K-component of Algol is the size of its Roche lobe, so that rules out one place to have the radio source. Second, Kippenhahn, Flannery and Ulrich have shown that a contact phase, with presumed large-scale mass loss probably occurs in the standard mass transfer episode. Therefore, one would want large amounts of mass to surround Algoltype systems. It is therefore crucial observationally to understand whether the events you see come from knots of gas which intercept fast particles from Algol. What is known about the location of Algol flare events with respect to Algol, and the location of one event with respect to another?

HJELLMING: Nothing. Only the relative size is known in some cases. 